\title{
UNA MIRADA AL ACCESO A LA UNIVERSIDAD EN EL MARCO DE LA POLÍTICA EDUCATIVA COLOMBIANA. 2010 a 2018
}

Ardila-Franco, Yurani Stella; Soto-Arango, Diana Elvira

UNA MIRADA AL ACCESO A LA UNIVERSIDAD EN EL MARCO DE LA POLÍTICA EDUCATIVA COLOMBIANA. 2010 a 2018

PANORAMA, vol. 14, núm. 26, 2020

Politécnico Grancolombiano, Colombia

Disponible en: http://www.redalyc.org/articulo.oa?id=343963784009

DOI: https://doi.org/10.15765/pnrm.v14i26.1487

Esta obra está bajo una Licencia Creative Commons Atribución-NoComercial-SinDerivar 4.0 Internacional. 
Artículos de investigación científica y tecnológica

\title{
UNA MIRADA AL ACCESO A LA UNIVERSIDAD EN EL MARCO DE LA POLÍTICA EDUCATIVA COLOMBIANA. 2010 a 2018
}

\author{
A LOOK AT ACCESS TO UNIVERSITY IN THE \\ FRAMEWORK OF COLOMBIAN EDUCATION \\ POLICY. 2010 to 2018
}

\author{
ACESSO À UNIVERSIDADE NO QUADRO DA \\ POLÍTICA EDUCATIVA COLOMBIANA. 2010 a 2018
}

Yurani Stella Ardila-Franco yurani.ardila@uptc.edu.co Universidad Pedagógica y Tecnológica de Colombia., Colombia Diana Elvira Soto-Arango dianaelvirasoto@gmail.com Universidad Pedagógica y Tecnológica de Colombia., Colombia

PANORAMA, vol. 14, núm. 26, 2020

Politécnico Grancolombiano, Colombia

Recepción: 11 Abril 2019

Aprobación: 19 Febrero 2020

DOI: https://doi.org/10.15765/ pnrm.v14i26.1487

Redalyc: http://www.redalyc.org/ articulo.oa?id=343963784009
Resumen: Este trabajo de investigación presenta los lineamientos que, a nivel mundial, ha trazado hacia la universidad el Banco Mundial, la OCDE, el BID y la Unesco, y que han impactado el acceso de los estudiantes a la universidad en lo referente al pago de matrículas y limitado el ingreso de la población de nivel socioeconómico bajo. Se analiza el periodo del año 2010 al año 2018, en el gobierno de Juan Manuel Santos, en Colombia. Se establece desde la historia social el respectivo análisis sociopolítico, a partir de la lectura de los planes decenales de educación, Conpes y normatividad correspondiente para la financiación de las universidades oficiales, los cuales se han concretado por medio de la constitución de programas como Ser Pilo Paga y ACCES, implementados por el Icetex. Este tipo de financiación estatal ha presentado rechazo por parte de la comunidad universitaria, porque ha resultado financiando a la universidad privada. Se realiza la revisión documental desde el acceso, cobertura, crédito, igualdad, educación técnica y tecnológica. Se concluye que se ha privatizado la universidad, lo que se demuestra por la desfinanciación de las instituciones del Estado (oficiales), y por la incidencia económica que trae a las familias los préstamos para el pago de la matrícula en ellas.

Palabras clave: Universidad, política educativa, acceso, cobertura, crédito educativo.

Abstract: The objective of the research was to present the guidelines that the World Bank, the OECD, the BID and Unesco have drawn towards the university and that have impacted the access of students to the university in terms of tuition and limiting the income of the population of low socio-economic level. The methodology used is based on qualitative research approaches based on a documentary review of the guidelines established at the international and national levels. The period from 2010 to 2018 of the government of Juan Manuel Santos in Colombia is analyzed. The respective sociopolitical analysis is established from the social history, from the reading of the plans of education, Conpes and corresponding regulations for the financing of the official universities, which have been materialized through the constitution of programs such as Ser Pilo Paga and ACCES, implemented through Icetex. This type of state funding has been rejected by the university community because it has financed the private university. The documentary review is carried out from access, coverage, credit, inequality, technical and technological education. It is concluded that the university has been privatized, which is demonstrated by the lack of financing for the (official) state university, and by the economic impact of loans for the payment of tuition at the university. 
Keywords: University, educational policy, access, coverage, educational credit.

Resumo: Este trabalho de pesquisa apresenta as diretrizes que, em nível global, foram elaboradas para a universidade pelo Banco Mundial, a OCDE, o BID e a Unesco e que tiveram um impacto no acesso dos estudantes à universidade em termos de pagamento de taxas e limitação da renda da população com um baixo nível sócio-econômico. $\mathrm{O}$ período de 2010 a 2018 do governo de Juan Manuel Santos na Colômbia é analisado. A respectiva análise sociopolítica é estabelecida a partir da história social, com base na leitura dos planos decenais de educação, Conpes e regulamentos correspondentes para o financiamento das universidades oficiais, que se materializaram através da constituição de programas como o Ser Pilo Paga e ACCES, implementados através do Icetex. Este tipo de financiamento estatal foi rejeitado pela comunidade universitária porque tem sido utilizado para financiar universidades privadas. A revisão documental é realizada do ponto de vista do acesso, cobertura, crédito, igualdade, educação técnica e tecnológica. Conclui-se que a universidade foi privatizada, o que é demonstrado, pela falta de financiamento da universidade estatal (oficial), e pelo impacto econômico com os empréstimos para o pagamento da matrícula universitária.

Palavras-chave: Universidade, política educativa, acesso, cobertura, crédito educativo.

\section{INTRODUCCIÓN}

El acceso a la educación superior es una preocupación constante en las poblaciones vulnerables y, por lo mismo, justifica el análisis en países como Colombia. A pesar de que la educación es un derecho, por mandato de la Constitución, sin embargo, en la realidad es un privilegio que depende en gran medida de dos circunstancias: el recurso económico para suplir los gastos de matrícula y sostenimiento y, por otra parte, de los saberes que trae el estudiante de su formación en la educación media. Desde la década de los sesenta se viene presentando una crisis financiera en la universidad estatal, con recomendaciones específicas sobre su autofinanciación, entre otros los informes de Atcon[2] y Rockefeller[3]. En la década de los 90, bajo la intervención del Banco Mundial (1995), se implementaron reformas y propuestas para el diseño de políticas, en especial para los países en desarrollo, con el ánimo de asegurar un uso eficiente de los recursos para el campo educativo y la universidad estatal. Uno de los cambios que se propusieron fue el desplazamiento de las funciones del gobierno, porque pasó de tener el control directo a la generación de políticas que promovieran cambios para las instituciones públicas y privadas, entre estas la vinculación de las familias en los gastos de la educación superior.

Así, para el Banco Mundial, el crecimiento económico puede generarse gracias al sector educativo, y este un componente que permite reducir la pobreza en los países en desarrollo, e insiste en la importancia de tener un menor gasto por estudiante para el manejo eficiente de recursos y así fortalecer la base financiera de la enseñanza pública, por ejemplo, no subvencionar gastos como el transporte o la alimentación, costos que deberían correr por cuenta del estudiante y su familia.

El impacto de las directrices internacionales generó consecuencias en la política nacional respecto a la forma en como se había manejado la financiación de la universidad. Para la universidad oficial, el presupuesto generado por parte del Estado era una parte vital para su sostenimiento, sin embargo, los costos de matrícula toman importancia desde que 
los organismos internacionales asumían que se debía involucrar a las familias como una forma de resolver parte de la crisis financiera de estas instituciones.

Con la Ley 30 de 1992[4], bajo el gobierno de César Gaviria y con el plan de desarrollo "La Revolución Pacífica (1990-1994), se implementó para las Instituciones de Educación Superior (IES), la libre competencia con relación a las instituciones privadas; se reconoció a las instituciones de carácter tecnológico, técnicas profesionales, universitarias, tecnológicas y universidades, y se dispuso la creación de programas en todos los niveles de formación. Sin embargo, la normatividad propuesta generó que la libre creación de programas fueran dominio de las instituciones privadas, direccionadas a los requerimientos económicos y políticos, lo cual motivó que la educación se organizara en términos de competitividad; en otras palabras, la incursión en el mercado educativo. Lo anterior condujo a un alza en las matrículas para las universidades privadas. La mayoría de estudiantes intentaban acceder a la universidad privada al no obtener un cupo en las estatales, provocando un endeudamiento de los alumnos y sus familias, porque tenían que acceder a un crédito para el ingreso a la universidad.

La elitización de la educación se visibilizó para los estudiantes de estratos bajos y que procedían de colegios públicos. Así que la posibilidad de acceso se reducía a la opción de adquirir un crédito educativo; sin embargo, estas opciones estaban precedidas por la poca capacidad adquisitiva, deficiente preparación desde el colegio y un bajo capital cultural de los estudiantes, circunstancias que fomentaron la desigualdad[5].

En el periodo de estudio del gobierno de Juan Manuel Santos (2010 a 2018), con sus planes de desarrollo en el primer periodo, "Prosperidad para Todos" (2010-2014), y en el segundo, "Todos por un Nuevo País" (2014-2018), cuyos objetivos eran construir una Colombia en paz, equitativa y educada, la realidad se impuso, la desigualdad se generó, ya que los estudiantes de estratos bajos tuvieron cada vez menos opciones para acceder a la universidad. En este sentido, se convirtió no en una opción, sino en una condición pertenecer a la educación técnica, tecnológica como el SENA o institutos de baja calidad. La política educativa cumplía con la premisa del acceso a la educación, sin embargo, la calidad de educación que se recibía dependía del estrato o condición socioeconómica de los jóvenes. Por otra parte, los nuevos programas de becas, con la modalidad de préstamos condonables, favorecieron a la universidad privada dado que se pagaba la matrícula al valor que estas instituciones la cobraban. Se evidenció otra desigualdad, pues a la universidad del estado se le daba en estas becas el valor de su matrícula, que indudablemente era mínima comparada con la pública, presentando una diferencia abismal que no solucionaba la crisis financiera de la institución oficial.

En la metodología, el enfoque de la presente investigación se enmarca en el método histórico hermenéutico propuesto por Cifuentes (2011), el cual no pretende controlar las variables, ni observar fenómenos en un entorno artificial, sino como enfoque de investigación implica el análisis 
de los hechos históricos en contextos sociales y políticos con el fin de interpretar, comprender y reconstruir situaciones, a partir del análisis de enunciados y afirmaciones que permitan la comprensión del fenómeno. Igualmente, se enmarca en la propuesta metodológica de la Sociedad de Historia de la Educación Latinoamericana (2017), y del grupo de investigación Historia y Prospectiva de la Universidad Latinoamericana, HISULA (2018).

El periodo de estudio seleccionado para la investigación inicia desde el año 2010 y va hasta el año 2018, periodo en el que para nuestro país se asientan una serie de acciones importantes en materia de acceso a la educación superior. De este modo, la investigación pretende tomar como referencia los planes decenales de educación de la época, la normatividad y Conpes en lo relacionado con las acciones en términos de acceso.

Desde el punto de vista histórico, se busca analizar las definiciones que se han instalado desde las políticas educativas en la concepción del acceso a la educación y cuál fue su afectación en la ciudadanía; desde el punto de vista social, revisar cuál fue la incidencia para los estudiantes, familias y comunidad académica con las disposiciones que se han adoptado en el funcionamiento de la universidad.

El marco conceptual está apoyado en la revisión de publicaciones de diferentes autores y teorías, en las que se encontraron diferentes visiones relacionadas con el acceso a la educación superior. Se han tomado fuentes primarias y secundarias con autores como Arrubla, Cuño, Soto, Gómez, Lazzarato, entre otros; también se revisaron enunciados en publicaciones de los organismos internacionales como OCDE, Banco Mundial, Unesco, CEPAL, y de los organismos nacionales como CESU, Icetex, MEN, información que se traza con los datos obtenidos por SPADIES para la construcción de los análisis que se pretende generar en la investigación. De igual manera, se estudió la normatividad correspondiente al objetivo del estudio.

En definitiva, esta investigación concluye que se ha privatizado la universidad oficial, lo cual se demuestra por la desfinanciación por parte del Estado, y por la incidencia económica en la población vulnerable, primero, en la dificultad para el acceso; segundo, si ingresa lo hace por medio de los préstamos para el pago de la matrícula y sostenimiento, con la consecuencia del endeudamiento de toda la familia.

\section{FUNDAMENTOS TEÓRICOS}

La desigualdad, de acuerdo con Cervera (2014), se expresaba en la calidad de los servicios, el prestigio, los profesores, pero además para los grupos poblacionales vulnerables se presentaba una "falsa" incursión en los ambientes educativos, en los que a nivel social continuaban siendo señalados como indeseables para el sistema. Las diferencias de clase, nivel socioeconómico, raza, entre otras, definían el tipo de institución al que el joven podía aspirar; el barrio, la comunidad, la familia, los amigos constituían el capital cultural que definía lo sucesivo de ese niño que se estaba formando. En este sentido, la educación, contribuía a una 
movilidad de la clase social; sin embargo, la educación privada, generaba la segregación escolar al ofrecer un servicio diferente al de la educación pública: era un servicio diferenciado que ratificaba el pago como una diferencia radical, exclusión que propiciaba la diferenciación del sector público y privado de un país.

El papel del Estado en relación a la superación de la desigualdad, además de la instalación de programas para el acceso, desde la perspectiva de Infante (2011), debía brindar la igualdad de oportunidades y la inclusión educativa para que se disminuyeran las desigualdades de orden social, étnico y de género, teniendo en cuenta las particularidades de cada estudiante o sus propias necesidades.

No era un secreto que los estudiantes de bajos recursos económicos estaban relacionados con un bajo nivel de competencias por su nivel socioeconómico y por proceder de colegios públicos, factores que hacían poco probable que tuvieran excelentes resultados en la prueba de acceso a la universidad. Respecto a esto, la OCDE (2012), afirmaba que la preparación de los estudiantes de estratos bajos no era garantía para el éxito de los estudiantes en la educación superior. Se referían factores como la edad promedio de los 16 años, y las deficiencias del sistema secundario en cuanto a la diferencia de las aspiraciones de los estudiantes y las habilidades que se adquirían en la educación secundaria. Dentro de este contexto reiteraba que estos estudiantes tenían menos probabilidades de ingresar a una universidad pública a partir del examen de admisión.

Por otro lado, el informe PISA del año 2009 evidenciaba la baja competencia de los estudiantes y las dificultades que esto generaba en sus estudios superiores. Existe una diferencia entre los conocimientos y competencias que se adquirían en el colegio y las competencias que se requerían para la educación superior; entonces, se evidenciaba una dificultad por parte de la educación pública respecto de la educación superior, pues suponía que la práctica estaba separada de los procesos académicos (OCDE, 2012). Por su parte, Moreno (2016, p.226), afirma que:

los alumnos nativos en familias nucleares y con mayor nivel socioeconómico y recursos en el hogar obtienen mayores rendimientos (...) Este hecho es especialmente significativo a la hora de señalar algunos aspectos que el sistema educativo debería tener en cuenta al garantizar la igualdad de oportunidades educativas.

Lo anterior reafirma que la familia de procedencia de los estudiantes determina las experiencias de aprendizaje, cuya afectación es la posición social del jóven.

En investigaciones realizadas por García (2007, p.71) citando a Téllez y Rodríguez (2003),

hay un 74\% de jóvenes entre 20-24 años que están fuera del sistema de educación superior en la región, siendo la mayoría de los excluidos jóvenes de menores recursos. De acuerdo con datos de CEPAL, el $60 \%$ de la población en edad universitaria, perteneciente a los sectores más pudientes de la población, se beneficia del $77 \%$ del gasto público total en educación superior, mientras el $40 \%$ restante de la población, 
perteneciente a los sectores menos pudientes, se beneficia apenas del $23 \%$ del gasto público total en educación superior.

De esta forma, se evidencian las disimilitudes y falta de oportunidades en el sector educativo y social en relación con la posición económica de las familias.

Las naciones que estaban en vía de desarrollo, al tener la presión por la demanda a la educación, generaban una apertura a la educación privada con fines de lucro. Según el Banco Mundial (2000), se buscaba priorizar la formación hacia la masa laboral con el propósito de satisfacer las necesidades de la economía en el marco del mercado. Así que la responsabilidad social del Estado pasaba a un segundo plano, de manera que el acceso estaba limitado por los ingresos, lo cual generaba exclusión para los estudiantes, aumentaba la importancia para el lucro y se disminuía la calidad.

Desde los planteamientos de la OCDE (2012), se buscaba que un gran número de estudiantes se encontrara matriculado en las IES, para esto se generó un aumento en los recursos para los préstamos, considerando el crédito educativo como una forma de disminuir la brecha social. En este sentido, la política garantizaba el acceso a la formación, sin embargo, la brecha se evidenciaba cuando la población con más recursos iba a la universidad y aquellos con menos recursos accedían a la formación técnica o tecnológica, siendo esta última -de manera paradójica- la que promocionaban los organismos internacionales para los países en desarrollo.

La apuesta por la educación técnica y tecnológica tenía su interés por la vinculación con la empresa privada, a propósito del crecimiento económico. La inversión en educación técnica y profesional era indispensable, en tanto los contenidos fueran relacionados con las competencias que facilitaran el mercado laboral y con la participación del sector privado, para que orientaran las demandas presentes y futuras de las empresas (OCDE, 2015).

Para el Banco Mundial (1995), la enseñanza superior orientaba su presupuesto a gastos no educacionales, como alimentación o vivienda subsidiados a los estudiantes, lo cual era una inversión ineficiente que representaba un gasto social regresivo. De esta manera, la participación de los estudiantes en los gastos a partir de los derechos de matrícula eran vitales para los recursos de la universidad; de acuerdo con el Banco, se podía mejorar la equidad a partir de subsidios para los estudiantes de bajos ingresos o programas de asistencia administrados por un organismo central; igualmente, se afirmaba que se debía permitir a las instituciones privadas fijar los derechos de matrícula, así como la autonomía para decidir sobre los estudiantes que podían recibir los programas académicos que ofrecían.

El Banco Mundial (1995), proponía en la reforma que los estudiantes de menos recursos accedieran a la educación técnica y tecnológica, pues se evidenciaba que los estudiantes privilegiados se encontraban en muy buenas escuelas secundarias de orden privado o universidades estatales gratuitas, mientras que los estudiantes con menos recursos tenían una 
educación de menor calidad en instituciones privadas de enseñanza terciaria.

Las afirmaciones del Banco correspondían a un pensamiento de orden neoliberal, cuando su interés principal se desviaba al pago de las matrículas; en otras palabras, que la universidad estuviera a la orden del lucro económico.

De acuerdo con el CESU (2013), la mayor parte de los estudiantes que accedían a los créditos eran de bajos recursos. De esta manera, trataba de demostrar que se tenía equidad y que el país ofrecía opciones de acceso. Sin embargo, en el mismo documento se afirmaba que: "La mayoría de las IES acreditadas son universidades, el $82 \%$. A la fecha del registro no hay ninguna institución técnica con acreditación institucional" (p.46). Sin embargo, la educación técnica era la que se recomendaba para la población de estratos socioeconómicos bajos. Por lo tanto, la educación superior seguía siendo inequitativa y excluyente para los estudiantes de familias pobres; la educación aún tenía un tono elitista y las tasas por el pago a la educación se relacionaban con el estatus.

La adquisición de un crédito educativo para acceder a la educación era un negocio importante para los bancos y entidades que financiaban la educación. De acuerdo con Lazzarato (2013), la deuda no era solo económica sino afectiva: el banco ayudaba a cumplir los "sueños" al apoyar al joven para el ingreso a la universidad. La adquisición de un crédito educativo reafirmaba la noción de que los ciudadanos eran responsables de su formación, y gracias al banco tenían la posibilidad de estudiar; los gastos de formación estaban en su totalidad a cargo de los estudiantes, lo que permitía liberar recursos que el Estado se apresuraba a transferir a las empresas y a los hogares más ricos, sobre todo mediante las reducciones de impuestos. La universidad se volvió un bien que se adquiría gracias al crédito educativo, y los estudiantes se convirtieron en sujetos de captura económica para las instituciones.

\section{DISCUSIÓN DE RESULTADOS}

En el informe de cierre del Plan Nacional Decenal 2006 - 2016, el Ministerio de Educación Nacional, MEN, concebía la educación como un derecho y un servicio público, que cumplía con una función social en concordancia con la Constitución Política Nacional y en compromiso con el Estado, la sociedad y la familia. Por su parte, la visión estaba orientada a que la educación era un derecho y un bien público de calidad, en condiciones de equidad e inclusión social por el Estado, y con la corresponsabilidad de la sociedad y la familia para la superación de la pobreza y la exclusión.

Entre los propósitos del plan estaban garantizar el derecho a la educación en condiciones de equidad para toda la población, hasta su articulación con la educación superior; la superación de la pobreza y la exclusión; la reconstrucción del tejido social y fomento de los valores democráticos; materialización de políticas por parte del Estado, que promovieran el aceso a oportunidades de progreso para los individuos. 
En relación con la cobertura en Educación Superior se buscaba que más bachilleres accedieran a este nivel educativo, así que para la cobertura de la educación técnica y tecnológica, para el año 2006 había 347.248 estudiantes matriculados, y en 2016 un total de 720.997 estudiantes.

En el 2002 inició el programa ACCES, cuyas características estaban referidas en el Conpes 3880 (2016, p.10):

Los principales componentes del programa son la equidad en el acceso a la educación superior mediante créditos educativos a los estudiantes de bajos recursos y mérito académico, la promoción de la formación técnica y tecnológica, el fortalecimiento de la formación doctoral, y el incremento de la capacidad institucional de las entidades sectoriales. Entre 2003 y 2015, ACCES ha beneficiado cerca de 414 mil estudiantes en programas de pregrado, de los cuales cerca del $80 \%$ son estrato 1 y 2 ".

Al continuar con las dificultades de acceso, en 2015 se constituyó el programa de crédito Tú Eliges, con el objetivo de ampliar las líneas de crédito de ACCES. Sin embargo, fueron muchos los estudiantes que se quedaron fuera del sistema.

Uno de los objetivos que se buscaba en el Plan Nacional de Desarrollo, "Más empleo, menos pobreza y más seguridad" (2010-2014, p.68):

Promover la innovación y el emprendimiento en todas las etapas de formación; desde la educación primaria, hasta la educación superior. En esta última, no solo fomentar la innovación y el emprendimiento empresarial entre estudiantes, sino también entre profesores e investigadores. Muchas grandes ideas nacen y mueren en las universidades, debido a la inadecuada alineación de incentivos, la falta de habilidades empresariales y de gestión de los propios profesores o investigadores universitarios, o la debilidad en las alianzas de las universidades con las empresas o con el sector público.

Así es que las instituciones de educación superior debían tener pertinencia en cuanto al emprendimiento, mediante la empresa desde los primeros años de estudio, a propósito del crecimiento, la sostenibilidad y la competitividad, así como el establecimiento de acuerdos entre universidad-empresa, siendo este uno de los principales propósitos del plan y quizá la apertura del sector privado a la educación.

Igualmente, el plan fomentó las competencias para la formación de capital humano, de acuerdo con las necesidades del sector productivo, siendo este último uno de los pilares del plan de desarrollo. Se llevó a cabo por medio de dos circunstancias; en primer lugar, el MEN propició incentivos para que el sector educativo hiciera transformaciones en su oferta en relación con los requerimientos de las empresas, en contenidos como competencias laborales, la formación para el trabajo y de la educación superior y, en segundo lugar, se buscó un aumento de cobertura de la educación técnica y tecnológica, fortaleciendo la enseñanza de competencias laborales específicas, el uso de las tecnologías de la información y las comunicaciones o los idiomas. Las acciones que se llevaron a cabo en materia de educación estaban direccionadas a la consolidación de la integración de la empresa con el sector educativo, en pro del crecimiento económico. 
Durante este gobierno se fomentó el Instituto Colombiano de Crédito Educativo y Estudios Técnicos en el Exterior (ICETEX), como institución promotora de programas para la promoción del acceso a la educación superior para los estratos más bajos, entre los cuales tomaron protagonismo el programa ACCES (Acceso con Calidad a la Educación Superior), y Ser Pilo Paga. De acuerdo con el Conpes 3880 (2016, p.7): "entre 2002 y 2015 la cobertura bruta en educación superior aumentó de $24,4 \%$ a $49,4 \%$ ", lo que indica un crecimiento aproximado a 20 puntos porcentuales durante los últimos 10 años, situación que se logró gracias a estrategias relacionadas con la promoción del financiamiento de la demanda de educación superior, esto con el apoyo del ICETEX, por el cual se incrementó la cobertura en el país y cuyas líneas de crédito han subsidiado a estudiantes de estratos 1,2 y 3 .

Según el Departamento Nacional de Planeación (2010), desde el año 2002 se instaló el proyecto ACCES, que buscaba la formación de capital humano para el mejoramiento de la competitividad en Colombia. La financiación correspondía a un $44 \%$ de un crédito con la banca multilateral, un 20,6\% de recursos que provienen del ICETEX y un 35,4\% con recursos de la Nación. En un principio el programa generó buenas críticas en relación con el acceso para la población vulnerable, en palabras de Sánchez y Márquez (2012, p.13):

este crédito ha tenido un impacto positivo en facilitar el acceso a la educación superior de jóvenes de estratos 1,2 y 3 y afirman que un porcentaje significativo de estudiantes no podría haber ingresado al sistema sin este programa, pues en el periodo $23,5 \%$ de estrato 3 . Comprendido entre 2003 y 2008, cerca de 130.000 jóvenes han sido beneficiados ( $19.7 \%$ de estrato $1,56,6 \%$ de estrato 2 y $23,5 \%$ de estrato 3 ).

Así, los jóvenes que ingresaron al sistema tuvieron la posibilidad para acceder a la educación superior.

Sin embargo, este crédito propuesto como política social para el acceso equitativo a la educación superior era una forma de privatizar la educación, según Gómez (2009), pues al estar respaldada con el Banco Mundial significaba que al gobierno se le dificultaba la ampliación de la cobertura en educación pública, entonces el estudiante subsidia a la educación privada mediante un crédito educativo. Además, las exigencias para el acceso a un crédito pasan por el análisis de la situación financiera del estudiante en relación con su estrato, que la institución elegida sea acreditada y el desempeño académico en la prueba Saber 11, situación que hacía difícil que un alumno en condición de desplazamiento, por ejemplo, lograra la aceptación de un crédito educativo. Por otra parte, al exigir que la universidad sea acreditada deja por fuera inmediatamente las instituciones de educación técnica y tecnológica, lo que genera una contradicción frente a lo que fomenta el gobierno y lo que exigía el Icetex. 


\begin{tabular}{|r|r|r|r|r|}
\hline \multicolumn{1}{|c|}{ AÑO } & NO RECIBIÓ & LARGO PLAZO & MEDIANO PLAZO & \multicolumn{1}{c|}{ ACCES } \\
\hline 1998 & 160817 & 1003 & 3889 & 1453 \\
\hline 1999 & 90805 & 1106 & 4371 & 1321 \\
\hline 2000 & 92028 & 1189 & 4380 & 2124 \\
\hline 2001 & 98726 & 1577 & 4208 & 3222 \\
\hline 2002 & 105224 & 1691 & 3925 & 4721 \\
\hline 2003 & 102608 & 1031 & 739 & 6810 \\
\hline 2004 & 98433 & 1372 & 489 & 11487 \\
\hline 2005 & 99300 & 1777 & 341 & 11058 \\
\hline 2006 & 102192 & 2309 & 279 & 13167 \\
\hline 2007 & 156980 & 4117 & 466 & 25038 \\
\hline 2008 & 157116 & 7985 & 346 & 21741 \\
\hline 2009 & 162959 & 7405 & 350 & 30225 \\
\hline 2010 & 167118 & 7552 & 326 & 26007 \\
\hline 2011 & 229495 & 8930 & 352 & 31288 \\
\hline 2012 & 219214 & 8312 & 318 & 31478 \\
\hline 2013 & 206775 & 7055 & 320 & 29714 \\
\hline
\end{tabular}

Tabla 1.

Tipo de crédito Icetex recibido, 1998 - 2013.

Fuente: SPADIES.

La gráfica muestra que había una gran cantidad de jóvenes que no recibieron un crédito por parte del Icetex. Por ejemplo, en el año 2013, 206.775 jóvenes no tenían un crédito del Icetex, frente a 29.714 que sí tenían un crédito ACCES, lo que quiere decir que el número de población con crédito era mucho menor; habría que preguntarse si la baja deserción se debía a la presión que generaba la deuda adquirida o a que en verdad los jóvenes deseaban continuar con sus estudios. Los estudiantes que obtuvieron un crédito ACCES presentaban una tasa de deserción del 35,6\%, mientras que los estudiantes que no tenían crédito alcanzaban una tasa de deserción del 52,1\%; además, los que tenían crédito ACCES presentaban mejores resultados académicos, lo que se traducía en "permanencia".

Respecto al acceso a la educación superior en Colombia, el grupo evaluador de la OCDE (2012), presentó dos consideraciones: en primer lugar, los estudiantes evaluados coinciden en que el nivel de educación alcanzado incidía en los ingresos futuros; respecto a la segunda consideración, se afirmaba que la universidad era la primera elección de los jóvenes siempre que la familia pudiera costear los gastos o tuviera la posibilidad de un crédito en el ICETEX; así la universidad tenía prestigio y la posibilidad de ingresos más altos que los técnicos o tecnólogos.

Desde las políticas se reconocía la importancia de fortalecer la educación técnica y tecnológica, a pesar de que se obtendrían menores ingresos. Del mismo modo, los jóvenes afirmaban que preferían las universidades públicas antes de las privadas por los costos y, por su parte, aquellos jóvenes que no tenían los recursos necesarios preferían estudiar en el SENA por la gratuidad, no obstante, no podían cubrir el número de solicitudes, siendo uno de cada siete solicitantes los que obtenía un cupo. 


\begin{tabular}{|c|c|c|c|}
\hline $\begin{array}{c}\text { Total de instituciones } \\
\text { activas ETDH }\end{array}$ & Certificado calidad & $\begin{array}{c}\text { Certificado calidad } \\
\text { vigente }\end{array}$ & $\begin{array}{c}\text { Porcentaje calidad } \\
\text { vigente }\end{array}$ \\
\hline 3.920 & 586 & 323 & $8,24 \%$ \\
\hline $\begin{array}{c}\text { Total de programas } \\
\text { activos ETDH }\end{array}$ & Certificado calidad & $\begin{array}{c}\text { Certificado calidad } \\
\text { vigente }\end{array}$ & $\begin{array}{c}\text { Porcentaje calidad } \\
\text { vigente }\end{array}$ \\
\hline 19.648 & 2.611 & 1.767 & $8,99 \%$ \\
\hline
\end{tabular}

Tabla 2.

Número de instituciones de Educación para el trabajo y desarrollo humano, 2020 Fuente: Ministerio de Educación Nacional.

A pesar de que la OCDE y el Banco Mundial apostaban por la educación técnica y tecnológica, la calidad de este tipo de instituciones era cuestionable. De acuerdo con la anterior tabla se puede afirmar que las instituciones de Educación para el Trabajo y Desarrollo Humano no disponían de un certificado de calidad en su totalidad; de 3.920 instituciones que existen, solo 586 tenían un certificado de calidad, y de 19.648 programas activos, solo 2.611 disponían de un certificado de calidad. En este orden de ideas, la formación técnica en el país no tenía la suficiente regulación para su funcionamiento, pero aun así se podía considerar como la "única" opción de formación para los estudiantes de estratos bajos.

Estrato

Fuente: SPADIES

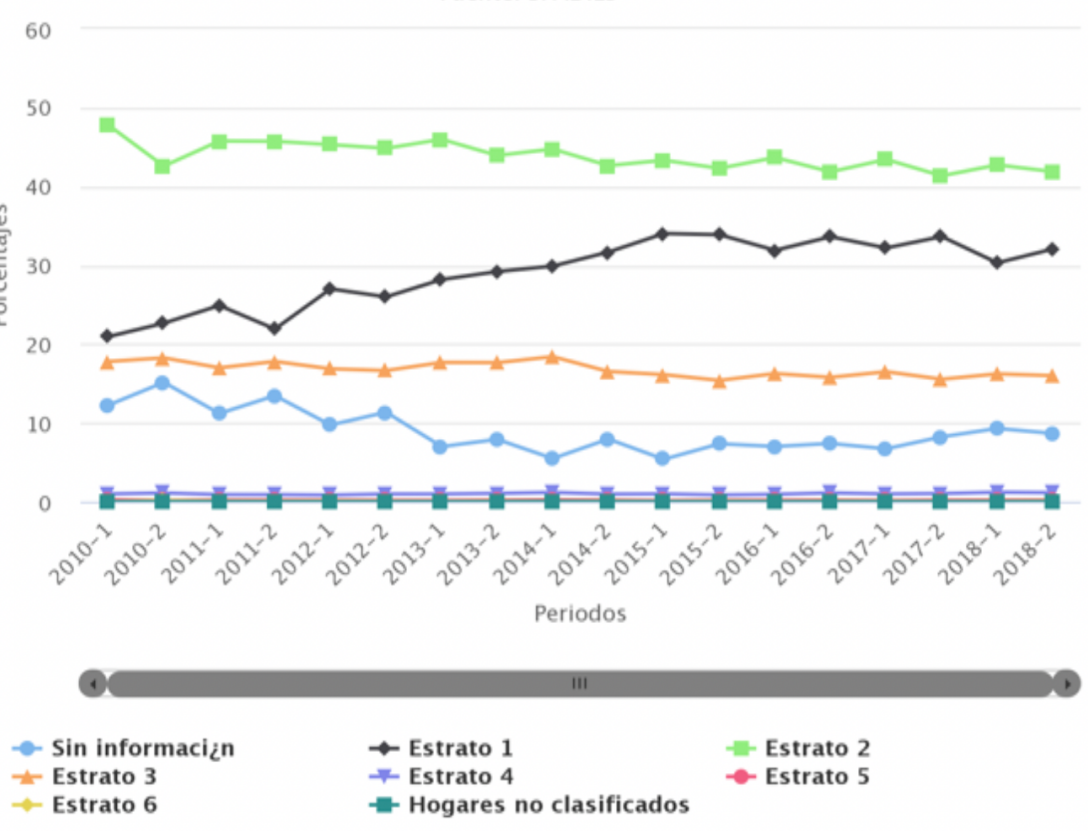

Gráfica 1.

Matrícula del SENA - 2010 - 2018

Fuente: SPADIES.

Se observa en la gráfica que los estratos 1 y 2 tenían un alto porcentaje de matrícula en el SENA; por ejemplo, para el segundo periodo de 2018 había matriculados un $43 \%$ de estudiantes pertenecientes al estrato 2 , 
frente a un $1 \%$ de estudiantes del estrato 5 . Con lo anterior podemos afirmar que era bastante marcada la estratificación de la formación; para los estratos altos no estaba por definición cursar estudios técnicos. En este caso, valdría la pena preguntarse si es que un estudiante de estrato bajo tuviera la posibilidad de acceder a una universidad, de igual manera elegiría estudiar en el SENA; de no ser así, el tema se reduce a un tema de recursos y oportunidades.

De acuerdo con las recomendaciones en materia de subvención, Sanguino (2017), cita a la OCDE afirmando que la financiación debía relacionarse con factores de calidad y generar la vinculación del SENA al sistema de educación superior. De esta manera, el gobierno se dispuso a diseñar un sistema de educación superior para darle la entrada al SENA y a instituciones de educación para el trabajo y el desarrollo humano, lo que quiere decir la modificación del esquema de titulación. De acuerdo con la OCDE, en Colombia había poca innovación, por tanto, era necesario trabajar con las demandas empresariales y las universidades, reforzar la relación Estado - empresa privada - IES, para innovaciones relacionadas con fusión entre instituciones de educación superior, redefinición de la estructura y duración de las carreras, reconocimiento de competencias adquiridas por fuera de la institución, es decir, que las empresas pudieran acreditar materias o experiencia.

En cuanto a los recursos, la OCDE manifestaba que el crecimiento de la matrícula no se lograba con la construcción de universidades públicas, se debía impulsar las carreras de ciclo corto y fortalecer las alianzas público privadas; así mismo, la gratuidad provocaba la inequidad en la educación superior. Lo anterior indicaba una clara tendencia de incorporar la empresa privada a la universidad. Los planteamientos internacionales sugerían que la universidad debía contemplar en sus planes o programas materias que tuvieran relación con las necesidades empresariales; además de fortalecer la universidad privada, no era factible la intención de abrir universidades estatales o generar un presupuesto para las mismas, de manera que las políticas públicas nacionales generaban acciones para incorporar las disposiciones de organismos como la OCDE.

A pesar de que la formación en Colombia estaba definida por cuatro tipos de instituciones de educación superior: universidades, instituciones universitarias, tecnológicas, técnicas, se optaba por esta última opción para los estudiantes con menos recursos, pues es la que de alguna forma prevalece para los estudiantes que provenían de la educación pública, básicamente por la falta de recursos y por el nivel de competencias que traían desde el colegio. Sin embargo, Gómez (2015) afirma que se le atribuye mucha importancia a la educación tradicional, de tal forma que se derivan jerarquías, con la universidad en la cúspide, y los otros tipos de instituciones más abajo, consideradas de segunda clase o categoría; estas instituciones tienen poca financiación, pues a pesar de que el Estado promueve esta formación, los recursos de los cuales disponen provienen de los ingresos de los estudiantes y sus familias.

En el periodo 2012 a 2014 se llevó a cabo un diálogo nacional, en el cual se presentó el documento de política pública para la 
educación superior, "Acuerdo por lo Superior 2034", en el cual se plantearon las acciones que debía asumir el país en relación con la educación superior. Los diez grandes temas que se abordaron fueron: educación inclusiva; calidad y pertinencia; investigación (ciencia, tecnología e innovación); regionalización; articulación de la educación media con la educación superior y la formación para el trabajo y el desarrollo humano (ETDH): hacia un sistema de educación terciaria; comunidad universitaria y bienestar; nuevas modalidades educativas; internacionalización; estructura y gobernanza del sistema y sostenibilidad financiera del sistema.

Dicho documento fue resultado de un trabajo de tres años, en los cuales se desarrollaron debates, se organizaron mesas temáticas en las regiones, se trabajó con expertos, se analizaron reformas educativas de otros países, además de otras acciones en torno a la discusión sobre la educación superior. El trabajo se organizó en cinco fases: inicio del diálogo a nivel nacional, análisis de propuestas y tendencias, organización de encuentros y talleres con el apoyo del CESU, y la deliberación, cuyo resultado fue la suma de esfuerzos y preocupaciones por parte de los ciudadanos para el mejoramiento y fortalecimiento de la educación superior en Colombia.

Entre los objetivos del Plan Nacional de Desarrollo (2014-2018), se buscaba que Colombia dirigiera sus esfuerzos al mejoramiento del acceso a los estudiantes desfavorecidos, por medio de la calidad de sus programas y generación de competencias. Para dar cuenta de los objetivos se estructuraron seis sistemas:

- El Sistema Nacional de Educacio\#n Terciaria (SNET), el cual contribuiría a modificar la educacio\#n y formacio\#n posterior a la educacio\#n media de acuerdo con pilares como la universidad y la educacio\#n y formacio\#n te\#cnica superior.

- La implementación del Marco Nacional de Cualificaciones (MNC), con el fin de clasificar los conocimientos, competencias y actitudes por niveles.

- El establecimiento de un Sistema Nacional de Acumulacio\#n y Transferencia de Cre\#ditos (SNATC), bajo los parámetros del Ministerio de Educación Nacional, con el fin de facilitar la movilidad entre instituciones educativas, niveles de formacio\#n y programas.

- El Sistema Nacional de Calidad de la Educacio\#n Superior (SISNACES), para apoyar a las instituciones de educacio\#n superior en el mejoramiento de la calidad, la eficiencia y pertinencia del sistema de aseguramiento de calidad.

- El Plan Maestro de Regionalizacio\#n, que buscaba ampliar la cobertura con calidad, con el ánimo de cerrar las brechas de acceso, equidad y calidad entre zonas rurales y urbanas, y entre las regiones o departamentos de Colombia.

- Formación de docentes a nivel de maestri\#as y doctorados (OCDE, 2016, p.272).

De acuerdo con el análisis y evaluación del Plan de Desarrollo 2014 - 2018 que hizo la Contraloría General de la República (2019), se reivindicaba en la importancia de la creación del Sistema Nacional de 
Educación Terciaria (SNET), que buscaba promover el aprendizaje, diciendo que era viable, puesto que había un $30 \%$ de la población que no estaba integrada a la educación superior. Así se afirma:

Esto responde a la necesidad de fortalecer ese nivel educativo frente al precario resultado en cuanto acceso y permanencia, ya que sus tasas de cobertura en el año 2013 fueron: bruta del 78,2\% (número total de matriculados/población total en edad escolar), y neta del $41,29 \%$, frente a la educación básica: cobertura bruta del 105,42\% y cobertura neta en el 88,8\% (número de matriculados en edad escolar/ población en edad escolar). (Contraloría General de la República, p.66)

En consideración, el SNET buscaba brindar las condiciones de competitividad que requería el país por medio de la educación universitaria y técnica, en disposición de la ampliación del acceso y cobertura necesarias, a pesar de la ausencia de condiciones para brindar formación a la mayor parte de la población.

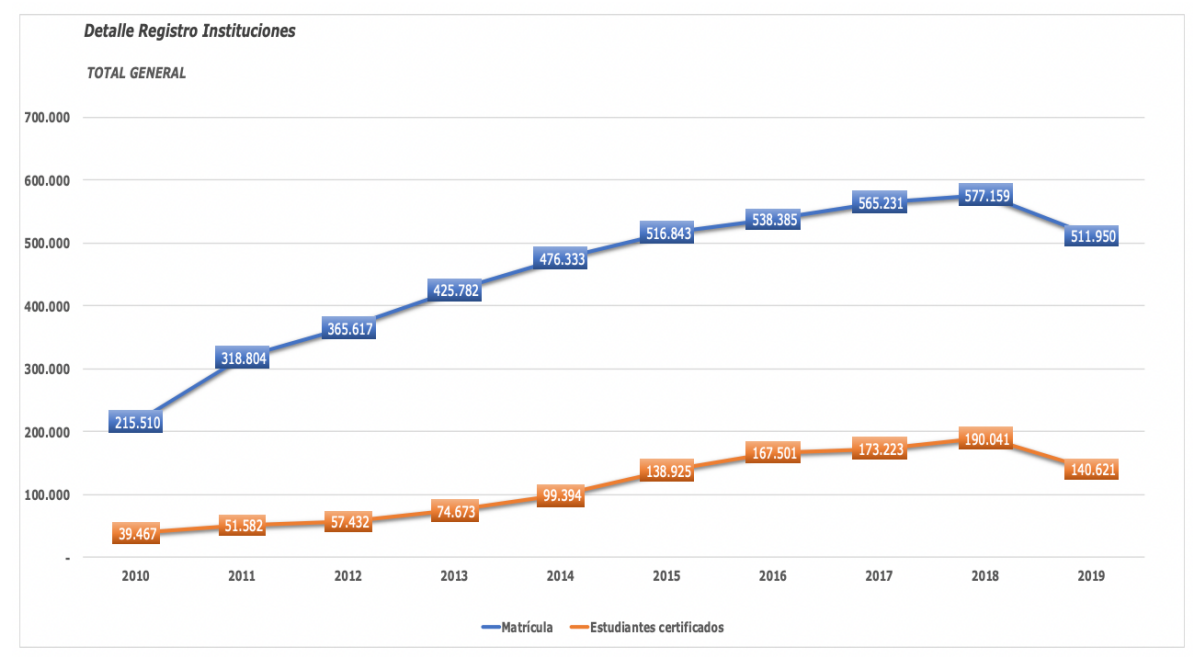

Gráfico 2.

Matrícula de Educación para el trabajo y el desarrollo humano 2010 - 2019. Fuente: Ministerio de Educación Nacional.

De acuerdo con la gráfica se puede determinar cómo hubo un aumento de la matrícula a partir de 2015, manteniéndose una cifra constante hasta el año 2019. Sin embargo, era muy inferior la cifra de estudiantes certificados; por ejemplo, para 2019, la cantidad de matriculados fue de 511.950 frente a 140.621 que lograron la certificación.

De acuerdo con cifras presentadas por el Conpes 3880 (2016, p.20):

Las metas definidas para educación superior por el PND implican alcanzar una tasa de cobertura bruta del $57 \%$ en 2018 , creando un total de 400 mil nuevos cupos en educación superior entre 2014 y 2018, de los cuales 150 mil serán en programas de formación técnica y tecnológica.

Más adelante se afirma que

Entre 2002 y 2015, la cobertura bruta en educación superior aumentó de $24,4 \%$ a 49,4\%. Mientras en 2002 un millón de estudiantes estaban matriculados en algún programa de educación superior, en 2015 esta cifra llegó a 2,3 millones de estudiantes, lo que refleja una clara expansión de la matrícula de este nivel (...) En total, el número de beneficiarios del Icetex 
ha aumentado de 55.583 en el año 2002 a 356.533 en 2015, y se ha logrado un incremento en el porcentaje de matrícula financiada, pasando de $6 \%$ a 16,5\% en el mismo periodo. (Conpes, 2016, p.22).

En relación con el puntaje de la prueba Saber 11 frente al acceso a la educación superior, el Conpes 3880 (2016, p.11) refleja que "según datos del Ministerio de Educación Nacional, el $23 \%$ de los jóvenes de estratos 1, 2 y 3 que obtuvieron los puntajes más altos de las pruebas Saber 11 en 2012 no habían accedido a educación superior dos años después de la prueba".

Con este panorama, en el año 2014 el MEN creó el programa Ser Pilo Paga, que cubría los costos de la matrícula y un subsidio de sostenimiento, dependiendo de la ciudad elegida. Para la primera convocatoria (2014-2015), se beneficiaron 10.141 jóvenes, y para la segunda (2015-2016), 12.715. Este programa mostró buenos resultados en cuanto al acceso a una universidad acreditada, sin embargo, con un notorio desbalance en el presupuesto público.

El programa se constituyó mediante el contrato de Fondos en Administración número 0077 de 2015, suscrito entre el Ministerio de Educación Nacional y el Icetex, en el cual se otorgaron créditos condonables para el acceso a la educación para los vinculados al programa Ser Pilo Paga. Según la OCDE (2016), el programa Ser Pilo Paga beneficiaba a una gran cantidad de estudiantes que no tenían la posibilidad de acceder a instituciones de educacio\#n superior de calidad, se caracterizaba por tener financiacio\#n pu\#blica y había una garantía por parte de las universidades para que los estudiantes finalizaran sus estudios. Sin embargo, el costo por estudiante superó las expectativas del gobierno; en el primer an\#o, el $85 \%$ de los estudiantes eligieron más universidades privadas que públicas, lo que produjo que el gobierno tuviera que generar más recursos. Así es que ingresaron menos estudiantes pobres y los que se retiraron quedaron con una deuda significativa.

De acuerdo con el Observatorio de la Universidad Colombiana (2018), en cuatro años con Ser Pilo Paga se beneficiaron 40.000 estudiantes, con una inversión de 3,5 billones de pesos desde 2015 hasta 2018, recurso que hubiera podido ser destinado para financiar todas las universidades públicas del país, las cuales tienen aproximadamente un millón de estudiantes. Para el año 2018, los aportes para financiar a las universidades estatales estaban en unos 3,6 billones de pesos, beneficiando a unos 600.000 estudiantes. Esto indicaba que dicho programa no tuvo el impacto adecuado, y más bien los dineros públicos seguían financiando a la universidad privada, mientras que la universidad oficial seguía desfinanciada, además de que el número de beneficiados no era representativo para una política de Estado en lo que respecta a la formación en educación superior.

Desde la percepción de Soto (2019, p.126):

Los análisis demostraron que con el presupuesto dado a las universidades privadas se hubiese ampliado la cobertura en las IES públicas y, por lo tanto, solucionado en parte su desfinanciación. Para el año 2018 el gobierno destinó un billón de pesos a cuarenta mil estudiantes de "Ser pilo paga" que fueron, en su mayoría, al presupuesto de las 
universidades privadas. Este dinero en la universidad pública financiaría seiscientos mil estudiantes.

Esto ratifica que el orden del presupuesto nacional estaba financiando a la universidad privada, a propósito del diseño de un programa que buscaba beneficiar a un grupo mínimo de estudiantes, en comparación con la cantidad de jóvenes que se encontraban por fuera del sistema.

Un fenómeno que se evidenció en este programa fue el aumento de matrículas, tal como sucedió en la Universidad de los Andes. Según la revista Semana (2017), los dineros del Estado a través del programa habían sido direccionados en su mayoría a la universidad privada, lo cual afectó el presupuesto para la universidad estatal. A continuación se presenta el incremento de matrículas de la Universidad de los Andes:

\begin{tabular}{|c|c|c|}
\hline & $\begin{array}{c}\text { COSTO DE } \\
\text { MATRÍCULA }\end{array}$ & $\begin{array}{c}\text { INCREMENTO } \\
\text { (\%) }\end{array}$ \\
\hline 2009 & $\$ 9.660 .000$ & $4.5 \%$ \\
\hline 2010 & $\$ 10.095 .000$ & $5.5 \%$ \\
\hline 2011 & $\$ 10.650 .000$ & $5.5 \%$ \\
\hline 2012 & $\$ 11.240 .000$ & $5.5 \%$ \\
\hline 2013 & $\$ 11.863 .000$ & $5.3 \%$ \\
\hline 2014 & $\$ 12.488 .000$ & $5.3 \%$ \\
\hline 2015 & $\$ 13.144 .000$ & $5.3 \%$ \\
\hline 2016 & $\$ 14.048 .000$ & $6.9 \%$ \\
\hline 2017 & $\$ 15.402 .000$ & $9.6 \%$ \\
\hline
\end{tabular}

Tabla 2.

Incremento de matrícula en licenciaturas Universidad de los Andes. Fuente: Revista Semana, 2017

De acuerdo con lo anterior, se puede notar cómo hay una estabilidad en el porcentaje anual de 5,5\% hasta el ingreso del programa, donde la matrícula subió considerablemente, casi un doble del año 2015 al año 2017.

De acuerdo con las 2Orillas (2018), universidades como los Andes, la Universidad del Norte, la Javeriana y La Salle fueron las instituciones que más obtuvieron recursos por parte del Ministerio de Educación a través del programa Ser Pilo Paga, con el $50 \%$ de su presupuesto. El diario informó que: 
la Universidad de los Andes, en particular, es la que más plata recibe del programa, $\$ 49.065^{\prime} 428.800$ en 2017 , por 1.869 pilos, que le representan un $10 \%$ de sus estudiantes de pregrado. Desde la tercera cohorte o selección, la Universidad del Norte en Barranquilla se convirtió en la preferida de los pilos. A finales de 2017 tenía 3.145 pilos, que le representaban el $24 \%$ de sus estudiantes de pregrado. Entre las públicas, la Universidad Nacional es la que mayor número de pilos ha recibido: 1.249 pilos, pero solo representa el 2,8 \% de sus estudiantes de pregrado. (2Orillas, 2018)

\begin{tabular}{|l|c|r|}
\hline \multicolumn{1}{|c|}{ IES acreditadas } & $\begin{array}{c}\text { TOTAL } \\
\text { beneficiarios } \\
\text { SPP 1 y SPP 2 }\end{array}$ & $\begin{array}{c}\text { Promedio valor } \\
\text { de matrícula por } \\
\text { estudiante }\end{array}$ \\
\hline Universidad de los Andes & 1.261 & $\$ 13.493 .075$ \\
\hline Fundación Universidad del Norte & 2.244 & $\$ 6.522 .506$ \\
\hline Pontificia Universidad Javeriana & 1.662 & $\$ 4.674 .133$ \\
\hline Universidad de la Salle & 1.701 & $\$ 4.460 .410$ \\
\hline Universidad de la Sabana & 1.050 & $\$ 9.304 .977$ \\
\hline Fundación Universidad Jorge Tadeo Lozano & 1.301 & $\$ 5.802 .266$ \\
\hline Universidad Santo Tomás & 481 & $\$ 4.884 .718$ \\
\hline Universidad Nacional de Colombia & 837 & $\$ 378.762$ \\
\hline Universidad Pedagógica y Tecnológica de Colombia & 405 & $\$ 1.046 .285$ \\
\hline
\end{tabular}

Tabla 3.

Listado de los giros que el Estado ha hecho a las universidades

beneficiadas con el programa Ser Pilo Paga - 2016.

Fuente: Observatorio de la Universidad Colombiana.

De acuerdo con lo anterior, se podría afirmar que la universidad privada había tenido mayor cantidad de recursos con este programa, contrario a lo que sucedía con la universidad pública, la cual obtienía la minoría de recursos.

De acuerdo con Lizarazo (2016), el número de estudiantes que ingresó a la universidad pública por el programa Ser Pilo Paga fue mucho menor que el de las universidades privadas: un 51,4 \% de las matrículas de los pilos fueron para siete universidades privadas, con un 98,4\% de la totalidad del presupuesto, es decir, $\$ 218.872 .001 .623$, y las oficiales, el $1,6 \%, \$ 3.558 .894 .334$, esto debido al bajo costo del semestre de las universidades públicas. Respecto al valor promedio de matrícula reconocido por cada estudiante del programa Ser Pilo Paga, en el 2016, en el primer puesto se destacó la Universidad de Manizales (\$5.408.697), y, en segundo lugar, la UPTC (\$1.046.285), en tercer lugar, la de Pereira (\$645.388.371), cuarto lugar la Nacional $(\$ 378.762)$ y quinto la UIS (\$411.972).

De acuerdo con el Observatorio de la Universidad Colombiana (2008), las universidades públicas presentaban un deficit presupuestal de unos 12.5 billones de pesos, lo que se podría evidenciar en la Universidad Nacional, donde recibía alrededor de 850 mil millones de pesos de transferencias del Estado para sostener a sus aproximadamente $55 \mathrm{mil}$ estudiantes, recursos que eran insuficientes; sin embargo, universidades como la Javeriana, la UniNorte y los Andes se veían beneficiadas con el dinero del Estado; la Universidad de los Andes tenía 2.500 estudiantes 
del programa Ser Pilo Paga, lo que equivalía a 15 millones de pesos por cada estudiante, es decir, 75 mil millones anuales, comparada con la universidad pública del Cesar que recibió 27 mil millones de pesos anuales por más de 20.000 estudiantes; si los recursos del programa se generaraban a la universidad pública se les podría subsidiar a 200.000 estudiantes la educación, pero al contrario se pretendía subsidiar a 40.000 estudiantes del programa.

Las políticas de educación superior, de acuerdo con Cuño (2019), mantuvieron el presupuesto público estable, sin embargo, se generó la disminución de la inversión por estudiante, a la par que la matrícula crecía significativamente. En Colombia, el mal manejo de los recursos públicos por parte de los gobiernos se evidenció con el programa Ser Pilo Paga, el cual podía ser denominado como un negocio educativo privado, ya que se financió con recursos públicos por cuatro años, donde se matricularon más de 40.000 jóvenes de bajos recursos en instituciones acreditadas de alta calidad en su mayoría privadas. El presupuesto estimado para el año 2015 fue de 155.000 millones de pesos colombianos, lo que en 2016 se convirtió en 374.000 millones de pesos colombianos, este dinero público se utilizó para financiar los costos de matrícula de esos negocios educativos privados.

A pesar del anterior escenario, la UNESCO (1998) se reafirmaba en que el acceso a la educación superior debía ser un derecho y más aún para la población con menos recursos:

Dentro de este marco es preciso asegurar el acceso a una educación superior de calidad y su permanencia en ella a las personas meritorias provenientes de los sectores sociales menos favorecidos; de ese modo las IES estarían contribuyendo al logro de mayor equidad social. Lograr este objetivo podría requerir políticas remediales o puntuales para subsanar las deficiencias que puedan tener determinados grupos sociales. (p.8)

A pesar de lo anterior, parece que la educación no se concibía como un derecho sino como un privilegio, pues aunque la política internacional promulgaba el acceso a la educación, se podía apreciar entre líneas que la formación universitaria era para quien podía pagar y la formación técnica y tecnológica era para aquellos que tenían pocos recursos.

Teniendo en cuenta lo mencionado, se podría afirmar que la estratificación de la educación sí existía, y que los jóvenes accedían a la educación en relación con los recursos que tuvieran, y la manera de suplir esta ausencia era a partir de los créditos educativos que se promulgaban desde las políticas educativas internacionales, adaptadas en la política nacional bajo el discurso de la equidad.

\section{CONCLUSIONES}

Los organismos internacionales con sus directrices están generando acciones para que la educación se oriente hacia el mercado. Es así que los gobiernos, a partir de la instalación de políticas, están procurando que la educación superior se constituya como un bien de consumo por el que se tenga que pagar, concibiéndolo como un servicio educativo, es 
decir, que solo una parte de la población podría pagar por este servicio. Por lo tanto, en Colombia, que siempre ha asumido y aplicado las políticas de organismos internacionales, trazó la cortina de humo al considerar la universidad como un bien público, y por lo tanto, financia en condiciones de igualdad a las instituciones privadas y oficiales, bajo la modalidad de concursos, dando como resultado -en primera instanciaque la universidad del Estado cada día ahonda la crisis financiera y, segundo, que solo unos pocos son los privilegiados para ser parte de la población universitaria.

Por otra parte, se generalizó en este periodo de estudio que la participación de los estudiantes y sus familias en el pago de la educación genera una apertura a la adquisición de créditos educativos, los cuales funcionan bajo la consigna de la equidad en la educación, pues se le da la opción al estudiante de bajos recursos de acceder a la educación mediante la búsqueda de un crédito. Por parte del Estado es el Icetex, con intereses altos, es el que se considera le va a permitir orientar su proyecto de vida. Sin embargo, la adquisición de un crédito trae consigo una deuda, cuyo pago genera presiones tanto para los estudiantes como para sus familias, pues el estar estudiando no es garantía de una rentabilidad futura.

Se destaca que en este periodo la ministra de educación privilegió la política de la educación técnica para los estratos socioeconómicos bajos, a través del SENA, puesto que era una opción más realista para los jóvenes con menores posibilidades, a pesar de que la calidad de este tipo de formación está en discusión en el contexto nacional. Este hecho pone en evidencia un tipo de exclusión educativa para los estudiantes de los estratos socioeconómicos más bajos. Lo relevante de esta formación superior radica en preparar a los estudiantes en un conocimiento técnico, el saber hacer, un aprendizaje que, si bien es importante, parece que estuviera dirigido a un grupo poblacional que no tiene posibilidad de la movilidad social.

Si bien en los planes de desarrollo se disponen medidas y acciones para el mejoramiento de la educación superior, aún falta desarrollar más esfuerzos para incrementar la cobertura para la formación superior, pues de cada 100 estudiantes que se gradúan del bachillerato solo ingresan a institutos o universidades unos 50 estudiantes, lo que indica que hay una gran cantidad de jóvenes en la informalidad, esto sin contar con aquellos que no logran terminar con éxito sus estudios.

Las líneas de crédito propuestas por el Icetex, en un principio parecen ser una buena opción para los estudiantes que no tienen recursos para acceder a la educación superior, sin embargo, en la práctica, los recursos de los créditos otorgados se direccionan en su mayoría a la universidad privada, lo cual genera que el estudiante de manera indirecta esté financiando este tipo de formación, tal como se puede evidenciar con el programa ACCES.

Los gobiernos al financiar la educación superior con los dineros públicos están privilegiendo a la universidad privada, como pasa con el programa Ser Pilo Paga. El programa en su inicio se diseñó para apoyar a los estudiantes destacados de bajos recursos, sin embargo, fue un programa 
en el cual la mayoría de los recursos se direccionaron a la universidad privada, además de instalarse como política de gobierno. Lo anterior indica la poca disposición de los diferentes gobiernos para fortalecer a la universidad estatal y el deficit fiscal que esta tiene, y que cada día se acrecienta con repercusiones sociales, porque ante los paros estudiantiles en la universidad pública, un número significativo de estudiantes pasan a la universidad privada, asumiendo deudas que las llevarán por años en su vida laboral.

En definitiva, el déficit de la universidad estatal aumenta la brecha para el acceso a esta institución. Y, en Colombia, este ingreso a la universidad significa un sueño colectivo familiar de mejorar las condiciones socioeconómicas, por medio de una profesión universitaria. Esta ilusión se frustra en el camino, en primera instancia por la deserción, y segundo, para los que logran la meta se encuentran con una realidad de desempleo y, por lo tanto, algunos se ubican laboralmente en otro oficio y un bajo porcentaje logra este objetivo familiar.

\section{REFERENCIAS}

1. Arrubla, M., \& Uribe, P. (2015). Ser pilo ¿paga o cuesta?: un análisis del programa de gobierno Ser Pilo Paga, a la luz del núcleo esencial del derecho fundamental a la Educación superior. Recuperado de: https://repository.e afit.edu.co/handle/10784/8254

2. Atcon, R. (1966). "La universidad latinoamericana. Propuesta para un enfoque integral del desarrollo social, económico y educacional en América Latina”. ECO Revista de la Cultura de Occidente.

3. Banco Mundial. (1995). La enseñanza superior: las lecciones derivadas de la experiencia. Recuperado de: http://documentos.bancomundial.org/curated/es/27421146832126 2162/La-ensenanza-superior-las-lecciones-derivadas-de-la-experiencia

4. Banco Mundial. (2000). La educación superior en los paises de desarrollo: peligros y promesas, grupo especial sobre educación superior y sociedad. Recuperado de: http://documentos.bancomundial.org/curated/es/63004146799850 5995/La-educacion-superior-en-los-paises-en-desarrollo-peligros-y-prom esas

5. Cervera, S., \& del Carmen, M. (2014). La jefatura de hogar en Cuba y América Latina un acercamiento a las desigualdades regionales y de género. Novedades en Población, 6(12), 1-29.

6. CESU. (2013). Acuerdo por lo Superior 2034. Recuperado de: http://aprende.colombiaaprende.edu.co/es/content/acuerdo-por-losuperior/w3-article-343920.php

7. Cifuentes, R. (2011). Diseño de proyectos de investigación cualitativa. Revista Tend Ret, (16), 2-4

8. Contraloría General de la República. (2019). Análisis y Evaluación Plan Nacional de Desarrollo 2014 - 2018 - Todos por un nuevo pais. Recuperado de: www.contraloria.gov.co 
9. Cuño, J. (2019). El espacio Iberoamericano del conocimiento en la perspectiva de transformación de la universidad en una microversidad. Revista Historia de la Educación Latinoamericana, RHELA, (32), 35-58.

10. Departamento Nacional de Planeación. (2010). Implantación acceso con calidad a la Educación Superior en Colombia ACCES fase II. Recuperado de: https://spi.dnp.gov.co/App_Themes/SeguimientoProyectos/Resum enEjecutivo/0020060060000.pdf

11. DNP. (2011). Plan Nacional de Desarrollo 2010 - 2014 Tomo I - Más empleo, menos pobreza y más seguridad. Recuperado de: https://legislacio n.vlex.com.co/vid/ley-expide-nacional-desarrollo-2010-336265973

12. Documento Conpes 3496. (2007). Recuperado de: https://www.dnp.gov.co /programas/desarrollo-social/subdireccion-de-educacion/Paginas/educa cion-superior.aspx

13. Documento Conpes 3880. (2016). Recuperado de: https://www.dnp.gov.c o/CONPES/documentos-conpes/Paginas/documentos-conpes.aspx

14. El Espectador. (2017). Aumento en las tarifas de U privadas y Ser Pilo Paga. Obtenida el 12 de julio de 2019, de https://blogs.elespectador.com/actualidad/conspirando-por-un-mun do-mejor/aumento-las-tarifas-u-privadas-pilo-paga

15. García, C. (2007). Financiamiento de la educación superior en América Latina. Revista Sociologías, (17), 50-101.

16. Gómez, N., Soto, D, \& Lima, J. (2018). Políticas y medición en ciencia y tecnología en la universidad colombiana. 1992-2014. Tunja: Universidad Pedagógica y Tecnológica de Colombia.

17. Gómez, V. (2009). Crédito educativo, acciones afirmativas y equidad social en la educación superior en Colombia. Revista de Estudios Sociales, (33), 106-117.

18. Gómez, V. (2015). La pirámide de la desigualdad en la educación superior en Colombia - Diversificación y tipología de instituciones. Colombia: Editorial Universidad Nacional de Colombia. 